\section{Article}

NAEEM, M. ${ }^{1,2}$

CHEEMA, Z.A. ${ }^{2}$

IHSAN, M.Z. ${ }^{1 *}$

HUSSAIN, Y. ${ }^{3}$

MAZARI, A. ${ }^{2}$

ABBAS, H.T. ${ }^{4}$

\title{
Allelopathic Effects of Different Plant Water EXTRACTS ON YIELD AND WEEDS OF WHEAT
}

\section{Efeitos Alelopáticos de Diferentes Extratos Aquosos de Plantas no Controle de Plantas Daninhas e no Rendimento do Trigo}

\begin{abstract}
Use of synthetic herbicides to control weeds poses serious threats to sustainable agricultural production around the globe. The phytotoxic effect of different plant water extracts (i.e., sorghum, sunflower and mulberry) against weeds has been previously studied; however, their combined effects at different rates are yet to be explored. In this study, mixtures of allelopathic water extracts of sorghum + sunflower and sorghum + sunflower + mulberry were sprayed each at $12,15,18$ and $21 \mathrm{~L} \mathrm{ha}^{-1}$ at 40 and 55 days after sowing in wheat to control wild oats (Avena fatua), little seed canary grass (Phalaris minor), lamb's quarters (Chenopodium album) and swine cress (Coronopus didymus). A synthetic herbicide Affinity 50 WP (Carfentrazoneethyl $0.75 \%$ + Isoproturon $50 \%$ applied at $1000 \mathrm{~g}$ a.i., ha ${ }^{-1}$ ) and a weedy check were kept as a control treatment. The increasing order of water extract dose 12-21 L ha-1 showed substantial reduction in total weed density and their dry biomass production of both narrow and broad-leaved weeds in all the treatment combinations. Mixture of sorghum + sunflower + mulberry water extracts each at $18 \mathrm{~L} \mathrm{ha}^{-1}$ applied at $40+55 \mathrm{DAS}$ resulted in an $87.14 \%$ decrease in total weed dry matter with a $19.5 \%$ increase in grain yield of wheat and higher net benefits of Rs. 100525 with $777.5 \%$ marginal rate of return. Nonetheless, application of sorghum + sunflower water extracts each at $12 \mathrm{~L} \mathrm{ha}^{-1}$ at $40+55$ DAS was more economical than the other treatments in terms of higher marginal rate of return of $799.82 \%$. Based on this study, it can be suggested that the use of allelopathic plant water extracts in a mixture may act as a potential weed control strategy in wheat.
\end{abstract}

Keywords: herbicide, weed management, allelopathy.

*Corresponding author:
<meeransaim@gmail.com

Received: May 11,2017

Approved: June 30, 2017

\section{Planta Daninha 2018; v36:e018177840}

Copyright: This is an open-access article distributed under the terms of the Creative Commons Attribution License, which permits unrestricted use, distribution, and reproduction in any medium, provided that the original author and source are credited.

\begin{abstract}
RESUMO - O uso de herbicidas sintéticos no controle de plantas daninhas representa uma séria ameaça à produção agrícola sustentável no mundo todo. $O$ efeito fitotóxico de diferentes extratos aquosos de plantas (por ex., sorgo, girassol e amora silvestre) sobre as plantas daninhas foi estudado anteriormente; no entanto, seus efeitos combinados em taxas diferentes ainda precisam ser explorados. Neste estudo, misturas de extratos aquosos alelopáticos de sorgo + girassol e sorgo + girassol + amora foram pulverizados cada a 12, 15, 18 e $21 \mathrm{~L} \mathrm{ha}^{-1}$ aos 40 e 55 dias após a semeadura em trigo para controle da aveia selvagem (Avena fatua), ervacabecinha (Phalaris minor), ançarinha-branca (Chenopodium album) e mastruço (Coronopus didymus). Um herbicida sintético Affinity 50 WP (carfentrazona-etílica 0,75\% + Isoproturão 50\% aplicado a $1000 \mathrm{~g}$ i.a., $\mathrm{ha}^{-1}$ ) e a capina foram mantidos como tratamento de controle. A ordem crescente da dose de extrato aquoso 12-21 L ha-1 mostrou uma redução substancial na densidade total de plantas daninhas e na produção de biomassa seca de plantas daninhas de folhas estreitas e de folhas largas em todas as combinações de tratamento. A mistura de extratos de
\end{abstract}

1 Cholistan Institute of Desert Studies, The Islamia University of Bahawalpur-63100, Pakistan; ${ }^{2}$ University of Agriculture Faisalabad-38040, Pakistan; ${ }^{3}$ Directorate of Agriculture Extension, Government of Punjab, Pakistan; ${ }^{4}$ Lasbela University of Agriculture, Water and Marine Sciences, Uthal-89250, Pakistan. 
sorgo + girassol + amora cada um a $18 \mathrm{~L} \mathrm{ha}^{-1}$ aos 40+55 DAS resultou em uma diminuição de 87,14\% na matéria seca total de plantas daninhas com 19,5\% de rendimento de trigo e maiores benefícios líquidos de Rs. 100525 com taxa marginal de retorno de 777,5\%. No entanto, a aplicação de extratos aquosos de sorgo + girassol cada um a12 $\mathrm{L} \mathrm{ha}^{-1}$ aos 40+55 DAS foi mais econômica que os demais tratamentos em termos de maior taxa marginal de retorno, de 799,82\%. Com base neste estudo, pode-se sugerir que o uso de extratos aquosos de plantas com efeito alelopático em mistura pode atuar como uma estratégia potencial de controle de plantas daninhas no trigo.

Palavras-chave: herbicida, manejo de plantas daninhas, trigo.

\section{INTRODUCTION}

The need for safe food production has developed steadily in the previous years as a result of awareness about food quality and environmental concerns because of agrochemicals used in agriculture (Bhandari, 2014). Worldwide huge crop losses have been found to result from heavy weed infestations. The losses associated with weeds in Pakistan exceed beyond Rs. 120 billion at a national level. A recent report has shown that the presence of weeds causes considerable yield losses (32-37\%) in wheat (Fahad et al., 2015). Zand et al. (2007) revealed that $30 \%$ grain yield losses are due to weed infestation. Farmers usually rely on quick and effective weed control measures by using synthetic herbicides which produce several environmental problems for human health as a result of indiscriminate use (Naeem et al., 2012; Ihsan et al., 2015).

Allelopathy is a natural, environmentally favorable approach that may prove to be a distinctive method for controlling weeds, improving crop yield, lessening our dependence on synthetic pesticides, and recovering the biological environment (Sodaeizadeh and Hosseini, 2012; Aslam et al., 2016). Many plant research studies proposed that allelopathy may prove to be an alternative to synthetic herbicides in terms of weed control (Jabran, 2017). Allelopathic crops such as sorghum (Weston et al., 2013; Won et al., 2013), sunflower (Alsaadawi et al., 2012; Jabran, 2017), brassica (Asaduzzaman et al., 2015), and mulberry (Haq et al., 2010; Jabran, 2017) are inhibitory to weeds. Foliar application of sorghum water extracts inhibited weeds in a wheat crop (Cheema and Khaliq, 2000 ) by $30-50 \%$, which was lower than the required level of weed control (80\%). Moreover, allelopathic activity does not appear to be due to the effect of a single compound; rather, it is more likely to be the result of the combination and interaction of many allelochemicals which appear to work in an additive or synergistic manner to reduce weed germination and growth (Ihsan et al., 2015). Allelopathic activities of different plants are selective, species-specific and concentration-dependent (Duke et al., 2007).

A diverse group of allelochemicals has been found in roots and aerial parts of sorghum i.e. phenolics, cyanogenic glycoside-dhurrin and sorgoleone as having weed suppressing ability (Weston et al., 2013). Sunflower contains terpenes and phenolic compounds as secondary metabolites. Likewise, the leaf extract of mulberry contains triterpenes, steroids, phenols and tannins as well (Díaz Solares et al., 2015). The sorghum metabolites extracted through methanol effectively disrupt the growth of broadleaf weeds (Won et al., 2013). It was also found that sunflower water extracts (100\%) inhibited total weed dry weight by $51 \%$ and increased wheat yield by $7 \%$ over control. The mulberry extracts inhibited the seedling growth of bermuda grass and interestingly, its foliar spray (100\%) significantly inhibited the growth of Bermuda grass and promoted wheat growth (Haq et al., 2010). Combined application of sorghum, sunflower and eucalyptus water extracts gave considerable weed suppression in wheat than their sole application (Cheema et al., 2003), possibly as a result of the manifestation of synergistic effects of differential phytochemicals in these species. The phytotoxic effect of sorghum, sunflower and mulberry on weed control in different crops have been previously studied; however their combined effects at different rates to suppress weed growth in wheat are yet to be explored. We assumed that when sorghum, sunflower and mulberry water extracts are tank mixed, they may show a synergistic phytotoxic effect in terms of winter weed suppression. 


\section{MATERIALS AND METHODS}

\section{Soil and site}

The present field study designed for exploring weed control with different crop aqueous extracts in wheat was conducted at the Agronomic Research Farm University of Agriculture, Faisalabad (31.25 $\mathrm{N}$ and $\left.73.09^{\circ} \mathrm{E}\right)$, Punjab, Pakistan during the winter of 2008-2009. The experimental soil belongs to the Lyallpur soil series (Aridisol-fine-silty, mixed, hyperthermic Ustalfic, Haplargid) in USDA classification and Haplic Yermosols in the FAO classification scheme.

\section{Crop husbandry}

The wheat variety Sehar-2006 was sown on a cultivated seed bed in $22 \mathrm{~cm}$ spaced rows using a single row hand drill on Dec 4, 2008 using seeds at $125 \mathrm{~kg} \mathrm{ha}^{-1}$. Fertilizers in the form of urea and diammonium phosphate were applied at 100 and $60 \mathrm{~kg} \mathrm{ha}^{-1}$, respectively. Half of the nitrogen and a full dose of phosphorous were applied at the time of sowing, while the remaining half of nitrogen was applied with the first irrigation. The first irrigation was performed at 20 days after sowing and subsequent irrigations were adjusted according to the climatic conditions and need of the crop.

\section{Experimental details}

For weed control, a mixture of sorghum, sunflower and mulberry water extracts was applied each at $12,15,18 \mathrm{~L} \mathrm{ha}^{-1}$. Synthetic herbicide Affinity $50 \mathrm{WP}$ (carfentrazone-ethyl $0.75 \%+$ isoproturon $50 \%$ applied at $1000 \mathrm{~g}$ a.i. $\mathrm{ha}^{-1}$ ) and a weedy check were maintained as control. The water extracts were prepared following the procedure by Cheema and Khaliq (2000) and were applied twice at 40 and 55 days after sowing (DAS). The label dose of Affinity 50 WP was applied at 30 DAS. The volume of the spray $\left(340 \mathrm{~L} \mathrm{ha}^{-1}\right)$ was determined after calibration by following the Ross and Lembi (1985) procedure. A knapsack hand-sprayer was used, fitted with a flat fan nozzle by maintaining a pressure of $207 \mathrm{kpa}$.

\section{Measurements}

The data on individual and total weed density and biomass in a unit area were recorded at 60 DAS with the help of a quadrate measuring $0.25 \mathrm{~m} \times 0.25 \mathrm{~m}$, randomly placed at two places in each experimental unit. The weeds were oven dried for 72 hours at $70{ }^{\circ} \mathrm{C}$ to get the dry weight. The wheat crop was harvested and threshed manually on the third week of April, 2009 from individual treatment plots; grain yield was weighed in kilograms and expressed as mega gram per hectare $\left(\mathrm{Mg} \mathrm{ha}^{-1}\right)$.

\section{Statistical analysis}

The data were analyzed statistically by using the statistical package MSTAT-C (Freed and Scott, 1986). Analysis of variance (ANOVA) was performed by using Fisher's analysis of variance technique while multiple comparisons among treatment means were made using the least significant difference (LSD) test at P $\leq 0.05$ (Steel et al., 1997).

\section{RESULTS AND DISCUSSION}

\section{Weed density}

The predominant weed flora in the field consisted of wild oat, little seed canary grass, swine cress, lamb's quarters and a few plants of field bindweed. All of the weed control treatments substantially inhibited density (68-87\%) of wild oats as compared with the weedy check (Table 1). There was maximum inhibition of both narrow (88-91\%) and broad leaf weeds (94-95\%) with the 
NAEEM, M. et al. Allelopathic effects of different plant water extracts on yield and weeds of wheat

Table 1 - Phytotoxic effect of allelopathic water extracts on density and dry biomass production of common wheat weeds

\begin{tabular}{|c|c|c|c|c|c|c|c|c|c|}
\hline \multirow{4}{*}{ Treatment } & \multirow{4}{*}{ Rate ha ${ }^{-1}$} & \multicolumn{4}{|c|}{ Grassy weeds } & \multicolumn{4}{|c|}{ Broadleaf weeds } \\
\hline & & \multicolumn{2}{|c|}{$\begin{array}{c}\text { Wild oat } \\
\text { (Avena fetua) }\end{array}$} & \multicolumn{2}{|c|}{$\begin{array}{l}\text { Little seed canary grass } \\
\quad(\text { Phalaris minor })\end{array}$} & \multicolumn{2}{|c|}{$\begin{array}{c}\text { Lamb's Quarters } \\
\text { (Chenopodium album) }\end{array}$} & \multicolumn{2}{|c|}{$\begin{array}{c}\text { Swine cress } \\
\text { (Coronopus didimus }\end{array}$} \\
\hline & & Density & $\begin{array}{c}\text { Dry } \\
\text { weight }\end{array}$ & Density & $\begin{array}{c}\text { Dry } \\
\text { weight }\end{array}$ & Density & $\begin{array}{c}\text { Dry } \\
\text { weight }\end{array}$ & Density & $\begin{array}{c}\text { Dry } \\
\text { weight }\end{array}$ \\
\hline & & $\left(\mathrm{m}^{-2}\right)$ & $\left(\mathrm{g} \mathrm{m}^{-2}\right)$ & $\left(\mathrm{m}^{-2}\right)$ & $\left(\mathrm{g} \mathrm{m}^{-2}\right)$ & $\left(\mathrm{m}^{-2}\right)$ & $\left(\mathrm{g} \mathrm{m}^{-2}\right)$ & $\left(\mathrm{m}^{-2}\right)$ & $\left(\mathrm{g} \mathrm{m}^{-2}\right)$ \\
\hline \multirow{2}{*}{ Weedy check } & \multirow{2}{*}{ - } & $14.00 \mathrm{a}$ & $13.09 \mathrm{a}$ & $18.67 \mathrm{a}$ & $23.52 \mathrm{a}$ & $9.00 \mathrm{a}$ & $4.05 \mathrm{a}$ & $13.00 \mathrm{a}$ & $8.45 \mathrm{a}$ \\
\hline & & - & - & - & - & - & - & - & - \\
\hline \multirow{2}{*}{ Sorghum + Sunflower each } & \multirow{2}{*}{$12 \mathrm{~L}$ at $40+55 \mathrm{DAS}$} & $4.50 \mathrm{~b}$ & $4.00 \mathrm{~b}$ & $5.53 \mathrm{~b}$ & $8.62 \mathrm{~b}$ & $2.67 \mathrm{~b}$ & $0.97 \mathrm{~b}$ & $4.67 \mathrm{~b}$ & $3.03 \mathrm{~b}$ \\
\hline & & $(68)$ & $(69)$ & $(70)$ & $(63)$ & $(70)$ & (76) & (64) & $(64)$ \\
\hline \multirow{2}{*}{ Sorghum + Sunflower + mulberry each } & \multirow{2}{*}{$12 \mathrm{~L}$ at $40+55 \mathrm{DAS}$} & $3.83 \mathrm{bc}$ & $3.26 \mathrm{bc}$ & $4.80 \mathrm{bc}$ & $7.30 \mathrm{bc}$ & $2.17 \mathrm{bc}$ & $0.97 \mathrm{~b}$ & $3.33 \mathrm{c}$ & $2.16 \mathrm{bc}$ \\
\hline & & (73) & $(75)$ & (74) & (69) & (76) & (76) & (74) & (74) \\
\hline \multirow{2}{*}{ Sorghum + Sunflower each } & \multirow{2}{*}{$15 \mathrm{~L}$ at $40+55 \mathrm{DAS}$} & $2.83 \mathrm{bcd}$ & $2.81 \mathrm{bc}$ & $4.67 \mathrm{bcd}$ & $6.86 \mathrm{bcd}$ & $1.5 \mathrm{~cd}$ & $0.67 \mathrm{bc}$ & $3.83 \mathrm{bc}$ & $2.49 \mathrm{~b}$ \\
\hline & & $(80)$ & $(79)$ & $(75)$ & $(71)$ & $(83)$ & $(83)$ & $(71)$ & $(71)$ \\
\hline \multirow{2}{*}{ Sorghum + Sunflower + mulberry each } & \multirow{2}{*}{$15 \mathrm{~L}$ at $40+55 \mathrm{DAS}$} & $3.00 \mathrm{bcd}$ & $2.67 \mathrm{bcd}$ & 3.83 cde & $5.70 \mathrm{bcd}$ & $1.17 \mathrm{de}$ & $0.62 \mathrm{bc}$ & $3.17 \mathrm{c}$ & $1.41 \mathrm{~cd}$ \\
\hline & & (79) & $(80)$ & (79) & (76) & $(87)$ & $(85)$ & (76) & $(83)$ \\
\hline \multirow{2}{*}{ Sorghum + Sunflower each } & \multirow{2}{*}{$18 \mathrm{~L}$ at $40+55 \mathrm{DAS}$} & $3.0 \mathrm{bcd}$ & $2.67 \mathrm{bcd}$ & $4.00 \mathrm{cde}$ & $6.61 \mathrm{bcd}$ & $1.17 \mathrm{de}$ & $0.22 \mathrm{c}$ & $2.83 \mathrm{~cd}$ & $1.19 \mathrm{~d}$ \\
\hline & & (79) & $(80)$ & $(79)$ & $(72)$ & $(87)$ & $(95)$ & $(78)$ & $(86)$ \\
\hline \multirow{2}{*}{ Sorghum + Sunflower + mulberry each } & \multirow{2}{*}{$18 \mathrm{~L}$ at $40+55 \mathrm{DAS}$} & $2.33 \mathrm{bcd}$ & $1.78 \mathrm{~cd}$ & $3.00 \mathrm{ef}$ & $5.39 \mathrm{bcd}$ & $0.67 \mathrm{de}$ & $0.27 \mathrm{c}$ & $2 \mathrm{de}$ & $1.19 \mathrm{~d}$ \\
\hline & & $(83)$ & $(86)$ & $(84)$ & $(77)$ & $(93)$ & (93) & $(85)$ & $(86)$ \\
\hline \multirow{2}{*}{ Sorghum + Sunflower each } & \multirow{2}{*}{$21 \mathrm{~L}$ at $40+55 \mathrm{DAS}$} & $2.67 \mathrm{bcd}$ & $2.37 \mathrm{bcd}$ & $3.33 \mathrm{de}$ & $5.63 \mathrm{bcd}$ & $0.67 \mathrm{de}$ & $0.29 \mathrm{c}$ & $2 \mathrm{de}$ & $1.08 \mathrm{~d}$ \\
\hline & & $(81)$ & $(82)$ & $(82)$ & $(76)$ & $(93)$ & (93) & $(85)$ & $(87)$ \\
\hline \multirow{2}{*}{ Sorghum + Sunflower + mulberry each } & \multirow{2}{*}{$21 \mathrm{~L}$ at $40+55 \mathrm{DAS}$} & $1.83 \mathrm{~d}$ & $1.63 \mathrm{~cd}$ & 2.83 ef & $4.90 \mathrm{~cd}$ & $0.5 \mathrm{e}$ & $0.22 \mathrm{c}$ & $1.17 \mathrm{ef}$ & $0.76 \mathrm{~d}$ \\
\hline & & $(87)$ & $(88)$ & $(85)$ & $(79)$ & (94) & $(95)$ & $(91)$ & $(91)$ \\
\hline \multirow{2}{*}{ Herbicide Affinity } & \multirow{2}{*}{$1000 \mathrm{~g}$ a.i. } & $1.67 \mathrm{~d}$ & $0.89 \mathrm{~d}$ & $1.67 \mathrm{f}$ & $3.37 \mathrm{~d}$ & $0.42 \mathrm{e}$ & $0.17 \mathrm{c}$ & $0.83 \mathrm{f}$ & $0.76 \mathrm{~d}$ \\
\hline & & $(88)$ & (93) & $(91)$ & $(86)$ & $(95)$ & (96) & (94) & $(91)$ \\
\hline LSD values at $5 \%$ probability level & & 2.469 & 1.863 & 1.420 & 3.604 & 0.978 & 0.581 & 1.147 & 0.908 \\
\hline
\end{tabular}

The values in parenthesis indicate the percent decrease in weed density and dry weight as compared to control; LSD: Least significant difference; Means in a column not sharing a letter in common differ significantly by Fisher's protected LSD at 5\% probability level.

synthetic herbicide. It was followed by allelopathic water extracts (WE) treatment viz. sorghum + sunflower + mulberry each at $21 \mathrm{~L} \mathrm{ha}^{-1}$ at 40+55 DAS and sorghum + sunflower + mulberry WE each at $18 \mathrm{~L} \mathrm{ha}^{-1}$ at 40+55 DAS (Table 1). These WE treatments (15-21 L ha $\left.{ }^{-1}\right)$, in the case of wild oat density, were statistically at par with each other while in case of little seed canary grass only the treatment (sorghum + sunflower + mulberry at 18-21 $\mathrm{L} \mathrm{ha}^{-1}$ ) was followed by the synthetic herbicide. The density of lamb's quarters by the WE treatments was almost similar in the treatment combination of sorghum + sunflower + mulberry at $15-21 \mathrm{~L} \mathrm{ha}^{-1}$ as well as in the water extract combination of sorghum + sunflower at 15-21 $\mathrm{L} \mathrm{ha}^{-1}$. However, maximum density inhibition in lamb's quarters $94 \%$ and swine cress $91 \%$ was achieved by sorghum + sunflower + mulberry each at $21 \mathrm{~L} \mathrm{ha}^{-1}$.

\section{Weed dry biomass production}

Weed dry biomass inhibition was similar as observed in case of their densities (Table 1). Both of the broad-leaved weeds lamb's quarters and swine cress were effectively inhibited by the synthetic herbicide by $95 \%$ and $96.3 \%$, respectively. Weed dry biomass production was inhibited in a similar way as density inhibition by the water extract treatments (Table 2). There was maximum total dry biomass inhibition $2.66 \mathrm{~g}(94.8 \%)$ over control in the herbicide as compared with the control treatment. Application of plant WE of sorghum + sunflower + mulberry each at $21 \mathrm{~L} \mathrm{ha}^{-1}$ caused $88 \%$ inhibition in total weed dry biomass over control. The WE treatment of sorghum + sunflower each at 15-21 L ha-1 and sorghum + sunflower + mulberry each at $21 \mathrm{~L} \mathrm{ha}^{-1}$ gave statistically equal reduction in weed dry biomass i.e. $77-87 \%$ (Table 2).

\section{Grain yield}

The adoption of an environmentally sound and sustainable weed control strategy resulted in enhancement of grain yield. Among the water extracts, there was maximum increase in grain 
Table 2 - The percent increase in wheat grain yield and decrease in total weed dry mass as influenced by different allelopathic water extracts

\begin{tabular}{|c|c|c|c|c|c|}
\hline Treatment & Rate ha ${ }^{-1}$ & $\begin{array}{c}\text { Total weed wry } \\
\text { weight inhibition }\end{array}$ & Grain yield & $\begin{array}{l}\text { Decrease in total } \\
\text { weed dry weight }\end{array}$ & $\begin{array}{c}\text { Increase in grain } \\
\text { yield }\end{array}$ \\
\hline & & $\left(\mathrm{g} \mathrm{m}^{-2}\right)$ & $\left(\mathrm{t} \mathrm{ha}^{-1}\right)$ & \multicolumn{2}{|c|}{$(\%)$} \\
\hline Weedy check & - & $51.18 \mathrm{a}$ & $2.93 \mathrm{~d}$ & - & - \\
\hline Sorghum + Sunflower each & $12 \mathrm{~L}$ at $40+55 \mathrm{DAS}$ & $14.31 \mathrm{~b}$ & $3.33 \mathrm{bcd}$ & 72.0 & 13.7 \\
\hline Sorghum + Sunflower + mulberry each & $12 \mathrm{~L}$ at $40+55 \mathrm{DAS}$ & $11.60 \mathrm{bc}$ & $3.27 \mathrm{bcd}$ & 77.3 & 11.6 \\
\hline Sorghum + Sunflower each & $15 \mathrm{~L}$ at $40+55 \mathrm{DAS}$ & $11.79 \mathrm{bc}$ & $3.03 \mathrm{~cd}$ & 77.0 & 3.4 \\
\hline Sorghum + Sunflower + mulberry each & $15 \mathrm{~L}$ at $40+55 \mathrm{DAS}$ & $9.14 \mathrm{~cd}$ & $3.27 \mathrm{bcd}$ & 82.1 & 11.6 \\
\hline Sorghum + Sunflower each & $18 \mathrm{~L}$ at $40+55 \mathrm{DAS}$ & $9.68 \mathrm{~cd}$ & $3.40 \mathrm{abc}$ & 81.1 & 16 \\
\hline Sorghum + Sunflower + mulberry each & $18 \mathrm{~L}$ at $40+55 \mathrm{DAS}$ & $6.58 \mathrm{~d}$ & $3.5 \mathrm{ab}$ & 87.1 & 19.5 \\
\hline Sorghum + Sunflower each & $21 \mathrm{~L}$ at $40+55 \mathrm{DAS}$ & $7.75 \mathrm{~d}$ & $3.27 \mathrm{bcd}$ & 84.9 & 11.6 \\
\hline Sorghum + Sunflower + mulberry each & $21 \mathrm{~L}$ at $40+55 \mathrm{DAS}$ & $6.11 \mathrm{de}$ & $3.40 \mathrm{abc}$ & 88.1 & 16 \\
\hline Herbicide Affinity $50 \mathrm{WP}$ & $1000 \mathrm{~g}$ a.i. & $2.66 \mathrm{e}$ & $3.78 \mathrm{a}$ & 94.8 & 29 \\
\hline LSD values at $5 \%$ probability level & & 3.782 & 0.424 & & \\
\hline
\end{tabular}

yield $\left(3.5 \mathrm{t} \mathrm{ha}^{-1}\right)$ with $19.5 \%$ increase from treatment that combined sorghum + sunflower + mulberry applied each at $18 \mathrm{~L} \mathrm{ha}^{-1}$ at 40-55 DAS over control. The WE treatments sorghum + sunflower each at $18 \mathrm{~L} \mathrm{ha}^{-1}$ and sorghum + sunflower + mulberry each at $21 \mathrm{~L} \mathrm{ha}^{-1}$ at 40+55 DAS were statistically at par with each other in terms of grain yield $(16 \%)$ over control. The yield increment by the application of other combinations of plant water extracts did not differ significantly. However, the highest grain yield $\left(3.78 \mathrm{t} \mathrm{ha}^{-1}\right)$ with $29 \%$ increase over control was achieved by herbicide application.

\section{Economic and marginal analysis}

All the treatments produced higher net benefits over control (weedy check) (Table 3). The highest net benefits (PKR 107375) were achieved with the synthetic herbicide. It was followed by the treatment sorghum + sunflower + mulberry each at $18 \mathrm{~L} \mathrm{ha}^{-1}$ at $40+55$ DAS. The marginal analysis revealed that the highest marginal rate of return $(779.82 \%)$ was achieved from the WE treatment sorghum + sunflower each at $12 \mathrm{~L} \mathrm{ha}^{-1}$ at 40+55 DAS (Table 4). It was followed by the

Table 3 - Economic analysis

\begin{tabular}{|c|c|c|c|c|c|c|c|c|c|c|c|}
\hline \multicolumn{12}{|c|}{ Treatment } \\
\hline Parameter & $\mathrm{T}_{1}$ & $\mathrm{~T}_{2}$ & $\mathrm{~T}_{3}$ & $\mathrm{~T}_{4}$ & $\mathrm{~T}_{5}$ & $\mathrm{~T}_{6}$ & $\mathrm{~T}_{7}$ & $\mathrm{~T}_{8}$ & $\mathrm{~T}_{9}$ & $\mathrm{~T}_{10}$ & Remark \\
\hline Grain yield & 2.93 & 3.33 & 3.27 & 3.03 & 3.27 & 3.4 & 3.5 & 3.27 & 3.4 & 3.78 & $\mathrm{Mg} \mathrm{ha}^{-1}$ \\
\hline Adjusted yield & 2.64 & 3.06 & 2.94 & 2.73 & 2.94 & 3.06 & 3.15 & 2.94 & 3.06 & 3.4 & $\begin{array}{l}\mathrm{Mg} \mathrm{ha}^{-1}(10 \% \text { reduction to bring at } \\
\text { farmers level) }\end{array}$ \\
\hline Gross income & 85800 & 99450 & 95550 & 88725 & 95550 & 99450 & 102375 & 95550 & 99450 & 110500 & Wheat price at PKR $32500 \mathrm{Mg}^{-1}$ \\
\hline Cost of herbicide & 0 & 0 & 0 & 0 & 0 & 0 & 0 & 0 & 0 & 2500 & $\begin{array}{l}\text { Affinity } 50 \text { WP (Carfentrazone-ethyl + } \\
\text { isoproturon) (PKR 1000/800 g) }\end{array}$ \\
\hline Cost of extracts & 0 & 267 & 400 & 333 & 500 & 400 & 600 & 467 & 700 & 0 & $\begin{array}{l}\text { Expenditure on preparation of extracts } \\
(100 / 18 \mathrm{~L})\end{array}$ \\
\hline Spray rent & 0 & 250 & 250 & 250 & 250 & 250 & 250 & 250 & 250 & 125 & PKR 125 spray $^{-1}$ \\
\hline Spray application cost & 0 & 1000 & 1000 & 1000 & 1000 & 1000 & 1000 & 1000 & 1000 & 500 & PKR 500 man $^{-1}$ day $^{-1}$ (one man-day ha ${ }^{-1}$ ) \\
\hline Total cost that vary & 0 & 1517 & 1650 & 1583 & 1750 & 1650 & 1850 & 1717 & 1950 & 3125 & PKR \\
\hline Net benefits & 85800 & 97933 & 93900 & 87142 & 93800 & 97800 & 100525 & 93833 & 97500 & 107375 & PKR ha $^{-1}$ \\
\hline Increase in net benefits (\%) & - & 14 & 9 & 2 & 9 & 14 & 17 & 9 & 14 & 25 & Compared with control \\
\hline
\end{tabular}

$\mathrm{T}_{1}=$ control (weedy check); $\mathrm{T}_{2}=$ Sorghum + sunflower water extracts each at $12 \mathrm{~L} \mathrm{ha}^{-1}$ at $40+55 \mathrm{DAS} ; \mathrm{T}_{3}=$ Sorghum + sunflower + mulberry water extracts each at $12 \mathrm{~L} \mathrm{ha}^{-1}$ at $40+55 \mathrm{DAS} ; \mathrm{T}_{4}=$ Sorghum + sunflower water extracts each at $15 \mathrm{~L} \mathrm{ha}^{-1}$ at $40+55 \mathrm{DAS} ; \mathrm{T}_{5}=$ Sorghum + sunflower + mulberry water extracts each at $15 \mathrm{~L} \mathrm{ha}^{-1}$ at $40+55 \mathrm{DAS} ; \mathrm{T}_{6}=$ Sorghum + sunflower water extracts each at $18 \mathrm{~L} \mathrm{ha}^{-1}$ at $40+55 \mathrm{DAS} ; \mathrm{T}_{7}=$ Sorghum + sunflower + mulberry water extracts each at $18 \mathrm{~L} \mathrm{ha}^{-1}$ at $40+55 \mathrm{DAS} ; \mathrm{T}_{8}=$ Sorghum + sunflower water extracts each at $21 \mathrm{~L}^{\text {ha }}{ }^{1}$ at $40+55 \mathrm{DAS} ; \mathrm{T}_{9}=$ Sorghum + sunflower + mulberry water extracts each at $21 \mathrm{~L} \mathrm{ha}^{-1}$ at $40+55 \mathrm{DAS} ; \mathrm{T}_{10}=$ Herbicide Affininty $50 \mathrm{WP}$ (Carfentrazone-ethyl $0.75 \%+$ Isoproturon $\left.50 \%\right)$ at $1000 \mathrm{~g}^{\mathrm{a} . \mathrm{i} .}$ ha ${ }^{-1}$; PKR $=$ Pakistani rupee $(1 \mathrm{US}$ dollar $=$ 105 PKR); DAS = days after sowing; a.i = active ingredient. 
Table 4 - Marginal analysis

\begin{tabular}{|c|c|c|c|c|c|c|c|}
\hline \multicolumn{2}{|r|}{ Extract/ herbicide } & \multirow{2}{*}{$\frac{\text { Rate } \mathrm{a}^{-1}}{-}$} & \multirow{2}{*}{$\begin{array}{c}\begin{array}{c}\text { Total variable } \\
\text { cost }\left(\mathrm{PKR} \mathrm{ha}^{-1}\right)\end{array} \\
0\end{array}$} & \multirow{2}{*}{$\begin{array}{c}\begin{array}{c}\text { Net benefits } \\
\left(\text { PKR ha }^{-1}\right)\end{array} \\
85800\end{array}$} & \multirow{2}{*}{$\frac{\text { Marginal cost }}{0}$} & \multirow{2}{*}{$\begin{array}{c}\begin{array}{c}\text { Marginal net } \\
\text { benefits }\end{array} \\
0\end{array}$} & \multirow{2}{*}{$\begin{array}{c}\begin{array}{c}\text { Marginal rate of } \\
\text { returns }(\%)\end{array} \\
0\end{array}$} \\
\hline $\mathrm{T}_{1}$ & control (weedy check) & & & & & & \\
\hline $\mathrm{T}_{2}$ & Sorghum + sunflower water extracts & $\begin{array}{c}\text { each at } 12 \mathrm{~L} \mathrm{ha}^{-1} \text { at } \\
40+55 \mathrm{DAS}\end{array}$ & 1516.67 & 97933 & 1517 & 12133.33 & 799.82 \\
\hline $\mathrm{T}_{4}$ & Sorghum + sunflower water extracts & $\begin{array}{c}\text { each at } 15 \mathrm{~L} \mathrm{ha}^{-1} \text { at } \\
40+55 \mathrm{DAS}^{2}\end{array}$ & 1583.33 & 87142 & 0 & 0 & $\mathrm{D}^{*}$ \\
\hline $\mathrm{T}_{3}$ & Sorghum + sunflower + mulberry water extracts & $\begin{array}{c}\text { each at } 12 \mathrm{~L} \mathrm{ha}^{-1} \text { at } \\
40+55 \mathrm{DAS}\end{array}$ & 1650 & 93900 & 0 & 0 & $\mathrm{D}$ \\
\hline $\mathrm{T}_{6}$ & Sorghum + sunflower water extracts & $\begin{array}{c}\text { each at } 18 \mathrm{~L} \mathrm{ha}^{-1} \text { at } \\
40+55 \mathrm{DAS}^{2}\end{array}$ & 1650 & 97800 & 0 & 0 & D \\
\hline $\mathrm{T}_{8}$ & Sorghum + sunflower water extracts & $\begin{array}{c}\text { each at } 21 \mathrm{~L} \mathrm{ha}^{-1} \text { at } \\
40+55 \mathrm{DAS}\end{array}$ & 1716.67 & 93833 & 0 & 0 & $\mathrm{D}$ \\
\hline $\mathrm{T}_{5}$ & Sorghum + sunflower + mulberry water extracts & $\begin{array}{c}\text { each at } 15 \mathrm{~L} \mathrm{ha}^{-1} \text { at } \\
40+55 \mathrm{DAS}\end{array}$ & 1750 & 93800 & 0 & 0 & $\mathrm{D}$ \\
\hline $\mathrm{T}_{7}$ & Sorghum + sunflower + mulberry water extracts & $\begin{array}{l}\text { each at } 18 \mathrm{~L} \mathrm{ha}^{-1} \text { at } \\
40+55 \mathrm{DAS}\end{array}$ & 1850 & 100525 & 333 & 2591.67 & 777.5 \\
\hline $\mathrm{T}_{9}$ & Sorghum + sunflower + mulberry water extracts & $\begin{array}{c}\text { each at } 21 \mathrm{~L} \mathrm{ha}^{-1} \text { at } \\
40+55 \text { DAS }\end{array}$ & 1950 & 97500 & 0 & 0 & D \\
\hline $\mathrm{T}_{10}$ & $\begin{array}{l}\text { Herbicide Affininty } 50 \text { WP (Carfentrazone- } \\
\text { ethyl } 0.75 \%+\text { Isoproturon } 50 \% \text { ) }\end{array}$ & at $1000 \mathrm{~g}$ a.i. ha ${ }^{-1}$ & 3125 & 107375 & 1175 & 6850 & 582.98 \\
\hline
\end{tabular}

PKR = Pakistani rupee $(1$ US dollar $=105$ PKR $)$; DAS = days after sowing; a.i = active ingredient. D* = Dominated because of fewer benefits or higher variable cost than the preceding treatment. Formula of Marginal rate of return $(\mathrm{MRR} \%)=\frac{\text { Change in net benefits }}{\text { Changein cost }} \times 100$.

treatment sorghum + sunflower + mulberry each at $18 \mathrm{~L}$ ha ${ }^{1}$ at $40+55$ DAS and label dose of herbicide with $775.5 \%$ and $582.98 \%$ MRR respectively, while all other treatments were uneconomical because of variable higher costs.

Several plant species produce a number of secondary metabolites, and some of these compounds play an imperative role in acting as protection machinery in the plant rhizosphere as allelopathic substances (Duke et al., 2000; Jabran, 2017). Aqueous extract treatments significantly inhibited the density and dry weights of weed species present at the experimental site. Weed density and dry biomass of wild oats, little seed canary grass, swine cress and lamb's quarters were significantly reduced by all the combinations of sorghum water extract with either of the extracts as with sunflower alone or sunflower + mulberry (Table 1). Inhibition in density and dry weights of both grassy and broad-leaved weeds substantially increased when increasing the rate of WE dose from 12 to $21 \mathrm{~L} \mathrm{ha}^{-1}$. Greater weed control at higher WE rates was may be due to increased concentration of allelopathic compounds present in sorghum such as m-coumaric acid, caffeic acids, gallic acid, protocatechuic acid, syringic acid, vanillic acid, p-hydroxybenzoic acid, p-coumaric acid, benzoic acid, ferulic acid (Nimbal et al., 1996; Weston et al., 2013; Won et al., 2013) in sunflower as á-naphthol, scopolin and annuionones (Anjum and Bajwa, 2005; Alsaadawi et al., 2012) and in mulberry as palmitic acid, ascorbic acid, gallic acid and vanillic acid (Haq et al., 2010). Weed density and dry matter production were reduced on a parallel basis with increasing dose of water extracts and also by combining three water extracts i.e sorghum + sunflower + mulberry instead of sorghum + sunflower (Table 1). These findings are supported by Naeem et al. (2016), who reported reduction in weed dry weight with sorghum and sunflower extracts by foliar spraying. Greater decrease in total weed dry biomass by WE treatments with increasing dose showed that higher concentration of allelochemicals may be more effective for weed biomass reduction. Dry matter accumulation reflects the growth behavior of a weed and gives a better indication of weed crop competition than weed density. Greater weed dry weight reflects more utilization of soil and environmental resources by the weeds at the expenses of crop growth. This relationship has been proved by an increase in grain yield with decreasing dry biomass. A similar set of results has also been reported by Cheema and Khaliq, (2000) in which $21 \%$ at $1: 10 \mathrm{w} / \mathrm{v}$ ratio of sorghum water extracts applied twice at 30 and 60 DAS increased wheat grain yield by $21 \%$ with decreases in weed density and dry weights by 44 and $49 \%$ over control, respectively. The maximum increase in grain yield over control in water extracts treatments was by sorghum + sunflower + mulberry each at $18 \mathrm{~L} \mathrm{ha}^{-1}$ instead of $21 \mathrm{~L} \mathrm{ha}^{-1}$ showed that at 
certain range of concentration, allelochemicals may have a negative effect on wheat yield. This assumption could be seen within this study, in which sorghum + sunflower + mulberry each at $12 \mathrm{~L} \mathrm{ha}^{-1}$ produced a similar amount of grain yield over control as achieved by sorghum + sunflower each at $21 \mathrm{~L} \mathrm{ha}^{-1}$ (Table 2). The net benefits gained by the application of sorghum, sunflower and mulberry WE each at $18 \mathrm{~L} \mathrm{ha}^{-1}$ gave fairly good net benefits (PKR 100525), followed by sorghum and sunflower WE each at $12 \mathrm{~L} \mathrm{ha}^{-1}$ with PKR 97933 (Table 4). However, the herbicide label dose gave the highest net returns PKR $107375 \mathrm{ha}^{-1}$. Yet, in terms of harmful effects of synthetic chemicals on the environment and in the context of organic agriculture (weed control,) the net benefits with water extracts are a landmark in the promotion of organic farming.

The results of the present research suggest a cumulative phytotoxic effect of sorghum, sunflower and mulberry in terms of weed control in wheat. The combination of potent allelopathic plant water extracts each at $18 \mathrm{~L} \mathrm{ha}^{-1}$ can be used as a weed management strategy in modern agriculture with an increase in wheat grain yield.

\section{REFERENCES}

Alsaadawi IS, Sarbout AK, Al-Shamma LM. Differential allelopathic potential of sunflower (Helianthus annuus L.) genotypes on weeds and wheat (Triticum aestivum L.) crop. Arch Agron Soil Sci. 2012;58(10):1139-48.

Anjum T, Bajwa R. A bioactive annuionone from sunflower leaves. Phytochemistry. 2005;66(16):1919-21.

Asaduzzaman M, Pratley JE, An M, Luckett DJ, Lemerle D. Metabolomics differentiation of canola genotypes: toward an understanding of canola allelochemicals. Front Plant Sci. 2015;5:765. doi: 10.3389/fpls.2014.00765

Aslam Z, Qamar-uz-Zaman, Ihsan MZ, Syed S, Fujii Y, Khaliq A et al. Efficacy of rice straw extracts in controlling weeds. Pakistan J Weed Sci Res. 2016;22(2):197-210.

Bhandari G. An overview of agrochemicals and their effects on environment in Nepal. Appl Ecol Environ Sci. 2014;2(2):66-73.

Cheema ZA, Khaliq A, Mubeen M. Response of wheat and winter weeds to foliar application of different plant water extracts. Pakistan J Weed Sci Res. 2003;9(1-2):89-97.

Cheema ZA, Khaliq A. Use of sorghum allelopathic properties to control weeds in irrigated wheat in semi arid region of Punjab. Agric Ecosys Envron. 2000;79(2-3):105-12.

Díaz Solares M, Cazaña Martínez Y, Pérez Hernández Y, Valdivia Ávila A, Prieto Abreu Marlene, Lugo Morales Y. Qualitative evaluation of secondary metabolites in extracts of Morus alba L.(mulberry) varieties and hybrids. Rev Cubana Plant Med. 2015;20(3):358-66.

Duke SO, Baerson SR, Rimando AM, Pan Z, Dayan FE, Belz RG. Biocontrol of weeds with allelopathy: conventional and transgenic approaches. In Novel biotechnologies for biocontrol agent enhancement and management. Springer, Dordrecht. 2007.p.75-85.

Duke SO, Dayan FE, Romagni JG, Rimando AM. Natural products as sources of herbicide, current status and future trends. Weed Res. 2000;40:99-111.

Fahad S. et al. Weed growth and crop yield loss in wheat as influenced by row spacing and weed emergence times. Crop Prot. 2015;71:101-8.

Freed RD, Scott DE. MSTAT-C. Crop and soil science. West Lafayette: Michigan State University, 1986.

Haq RA, Hussain M, Cheema ZA, Mushtaq MN, Farooq M. Mulberry leaf water extract inhibits bermudagrass and promotes wheat growth. Weed Biol Manage. 2010;10(4):234-40.

Ihsan MZ, Khaliq A, Mahmood A, Naeem N, El Nakhlawy F, Alghabari A. Field evaluation of allelopathic plant extracts alongside herbicides on weed management indices and weed-crop regression analysis in maize. Weed Biol Manage. 2015;15(2):78-86.

Jabran K. Manipulation of allelopathic crops for weed control. Dordrecht: Springer: 2017. 
Naeem M, Mahmood A, Ihsan MZ, Daur I, Hussain S, Aslam Z et al. Trianthema portulacastrum and Cyperus rotundus interference in maize and application of allelopathic crop extracts for their effective management. Planta Daninha. 2016;34(2):209-18.

Naeem M, Cheema ZA, Azraf-ul-Haq Ahmad, Abdul Wahid A, Kamaran M, Arif M. Weed dynamics in wheat-canola intercropping systems [online]. Chilean J Agric Res. 2012;72(3):434-9.

Nimbal CI. Phytotoxicity and distribution of sorgoleone in grain sorghum germplasm. J Agric Food Chem. 1996;44(5):1343-7.

Ross MA, Lambi CA. Applied weed science. Minneapolis: Burgess Publishing; 1985. p.133-9.

Sodaeizadeh H, Hosseini Z. Allelopathy an environmentally friendly method for weed control. InTechOpen; 2012.

Steel RGD, Torrie JH, Dickey DA. Principles and procedures of statistics: a biometric approach. $3^{\text {rd }}$ ed. New York: McGraw Hill Book; 1997.

Weston LA. Sorghum allelopathy-from ecosystem to molecule. J Chem Ecol. 2013;39(2):142-53.

Won OJ, Uddin MR, Park KW, Pyon JY, Park SU. Phenolic compounds in sorghum leaf extracts and their effects on weed control. Allelopathy J. 2013;31(1):147-56.

Zand E, Ali Baghestani M, Soufizadeh S, Eskandari A, PourAzar R, Veysi M et al. Evaluation of some newly registered herbicides for weed control in wheat (Triticum aestivum L.) in Iran. Crop Prot. 2007;26(9):1349-58. 